Methods: Patients with a crystal-proven diagnosis of CPPD and age- and sexmatched disease-controls were prospectively enrolled in this cross-sectional, monocentric, case-control study. All subjects underwent a bilateral US examination of 9 hyaline cartilages $(\mathrm{HC}), 6$ fibrocartilages $(\mathrm{FC}), 5$ tendons, 1 joint recess and 1 ligament as follows: shoulder (glenoid $\mathrm{FC}$, humeral $\mathrm{HC}$ and acromioclavicular FC), elbow (humeral $\mathrm{HC}$ and triceps tendon), wrist (triangular FC, scapho-lunate ligament, volar recess of the radio-lunate joint), hand ( $\mathrm{HC}$ of the metacarpophalangeal joints from $2^{\text {nd }}$ to $5^{\text {th }}$ finger), hip (acetabular FC and femoral $\mathrm{HC}$ ), knee (femoral condyles' HC, meniscal FC, patellar and quadriceps tendons), ankle (talar HC, Achilles tendon and plantar fascia). US assessment was carried-out by a rheumatologist blinded to clinical data. CPP deposits were identified as presence/absence, according to the OMERACT definitions [1]. Results: Ninety-five patients were enrolled: 45 CPPD patients (age: $72 \pm 10.6$ years, disease duration: $5.6 \pm 7.8$ years, female/male ratio: 1.3 ) and 50 age- and sex-matched disease-controls (18 with rheumatoid arthritis, 13 with osteoarthritis, 10 with psoriatic arthritis and 9 with gout).

The FC of the medial and lateral meniscus were the most frequently involved targets of CPP deposits in cases ( $81.8 \%$ and $77.3 \%$ of patients, respectively), followed by the triangular $\mathrm{FC}$ of the wrist $(68.2 \%)$, the $\mathrm{HC}$ of the femoral condyles $(54.5 \%)$, the scapho-lunate ligament $(52.3 \%)$ and the acetabular FC $(50.0 \%)$ In all these anatomical targets, US findings indicative of CPP deposits were detected in a significantly higher percentage of cases than controls $(p<0.01)$. The US scanning protocols that showed the best balance between sensitivity and specificity, the most sensitive and the most specific were shown in Table 1.

Table 1. Diagnostic performances of different US scanning protocols

\begin{tabular}{lcccc}
\hline Anatomical targets & SE & SP & LH+ & LH- \\
\hline Knee meniscal FC and wrist triangular & 0.86 & 0.86 & 6.35 & 0.13 \\
FC & $(0.76-0.96)$ & $(0.73-0.94)$ & $(3.17-12.72)$ & $(0.06-0.30)$ \\
Knee meniscal FC, wrist triangular FC & 0.93 & 0.82 & 5.19 & 0.08 \\
$\quad$ and hip acetabular FC & $(0.82-0.99)$ & $(0.69-0.91)$ & $(2.85-9.42)$ & $(0.03-0.24)$ \\
Hip acetabular FC, knee femoral & 0.67 & 0.96 & 16.67 & 0.35 \\
condyles' HC and & $(0.51-0.80)$ & $(0.86-0.99)$ & $(4.22-65.82)$ & $(0.23-0.53)$
\end{tabular}

wrist scapho-lunate ligament

LH: likelihood ratio, SE: sensitivity, SP: specificity, US: ultrasonography.

In all US scanning protocols, each anatomical target was assessed bilaterally.

Figure 1 includes representative pictures showing CPP crystal deposits in different anatomical targets.

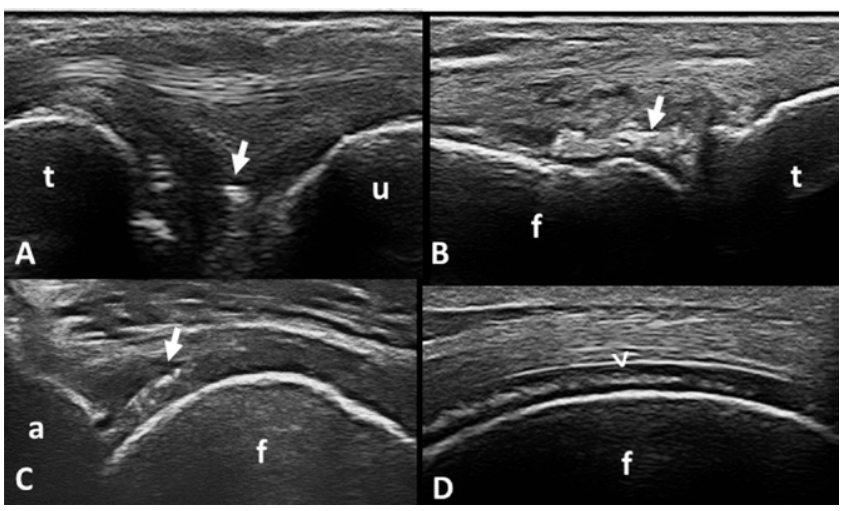

A: Wrist, longitudinal scan of the triangular FC complex.

$\mathrm{B}$ : Knee, longitudinal scan of the lateral meniscus.

C: Hip, longitudinal scan of the acetabular labrum FC,

D: Knee, longitudinal scan of the medial femoral condyle's HC.

Arrows: CPP crystal deposits at FC, arrowhead: CPP crystal deposits at $\mathrm{HC}$.

Conclusion: Our results suggest that bilateral US assessment of knee, wrist and hip provided acceptable sensitivity and specificity for diagnosing CPPD. REFERENCES:

[1] Filippou G, et al. Identification of calcium pyrophosphate deposition disease (CPPD) by ultrasound: reliability of the OMERACT definitions in an extended set of joints-an international multiobserver study by the OMERACT Calcium Pyrophosphate Deposition Disease Ultrasound Subtask Force. Ann Rheum Dis. 2018;77:1194-9.

Disclosure of Interests: Edoardo Cipolletta: None declared, Jacopo Di Battista: None declared, Walter Grassi Speakers bureau: Walter Grassi has received speaking fees from AbbVie, Celgene, Grünenthal, Pfizer and Union Chimique Belge Pharma., Emilio Filippucci Speakers bureau: Emilio Filippucci. has received speaking fees from AbbVie, Bristol-Myers Squibb, Janssen-Cilag, Novartis, Pfizer, Roche and Union Chimique Belge Pharma.

DOI: 10.1136/annrheumdis-2021-eular.496

\section{OP0206 \\ ASSOCIATION BETWEEN FEMALE REPRODUCTIVE FACTORS AND GOUT: NATIONWIDE POPULATION- BASED COHORT STUDY OF 1 MILLION POSTMENOPAUSAL WOMEN}

Y. Eun ${ }^{1}$, I.Y. Kim², K. D. $\mathrm{Han}^{3}$, K. Lee ${ }^{3}$, S. Y. Kang ${ }^{1}$, S. Lee ${ }^{1}$, H. S. Cha ${ }^{1}$, E. M. Koh' ${ }^{1}$, J. Lee ${ }^{1}$, H. Kim ${ }^{4} .{ }^{1}$ Samsung Medical Center, Sungkyunkwan University School of Medicine, Department of Medicine, Seoul, Korea, $c$ of (South Korea); ${ }^{2}$ National Police Hospital, Department of Medicine, Seoul, Korea, Republic of (South Korea); ${ }^{3}$ Soongsil University, Department of Statistics and Actuarial Science, Seoul, Korea, Republic of (South Korea); ${ }^{4}$ Samsung Medical Center, Sungkyunkwan University School of Medicine, Department of Medical Humanities, Seoul, Korea, Republic of (South Korea)

Background: Previous studies have shown that the incidence of gout differs according to gender, and its risk factors also differ according to gender. However little research has been done on the association between reproductive factors and gout.

Objectives: Our study attempted to determine whether there is an association between reproductive factors and the incidence of gout in a large nationwide population-based cohort of postmenopausal women.

Methods: Postmenopausal women aged 40-69 who participated in national health screenings in 2009 were included in the study. Subjects who had been diagnosed with gout prior to medical examination were excluded from the study, and a total of 1,076,378 women were included in the study. Outcome was the occurrence of gout which was defined as a case of two outpatient visit or one hospitalization for gout using the ICD-10 code of gout (M10) in the claim database. The Cox proportional hazard model was used for the analysis, and stratified analysis according to the body mass index (BMI) and chronic kidney disease (CKD) was performed.

Results: Mean follow-up duration was 8.1 years, and incident cases of gout were 64,052. Later menarche (adjusted HR 1.10, 95\% Cl 1.02-1.19 in >16 years compared with $\leq 12$ years), earlier menopause (adjusted HR 1.12 in $<40$ years, 1.06 in 40-45 years, 1.03 in 45-50 years, compared with 50-55 years), and shorter reproductive span (adjusted HR 1.10 in <30 years, 1.06 in 30-35 years, compared with $\geq 40$ years) were associated with a high risk of gout. No association between parity and gout incidence was observed. Use of oral contraceptives (OC; adjusted HR 1.03 in <1 year, $1.05 \geq 1$ year, compared with non-user) and hormone replacement therapy (HRT; adjusted HR 1.16 in $<2$ years, 1.16 in 2-5 years, 1.18 in $\geq 5$ years, compared with non-user) were associated with an increased risk of gout. The association between reproductive factors and gout remained in the trend without statistical significance in the low BMI group, but not in the high BMI group. The effects of use OC and HRT on gout were not significant in the CKD group.

Conclusion: Shorter exposure to endogenous estrogen was associated with a high risk of gout. Conversely, exposure to exogenous estrogen such as $\mathrm{OC}$ and HRT was associated with an increase in gout risk. This association was not significant in subjects with high BMI or CKD.

Disclosure of Interests: None declared

DOI: 10.1136/annrheumdis-2021-eular.4169

\section{OP0207 ASSOCIATION BETWEEN DEMENTIA AND GOUT: A NATIONWIDE POPULATION-BASED STUDY}

J. Kim ${ }^{1}$, H. M. P. Park ${ }^{2}$, D. H. Yim ${ }^{3}$, I. A. Choi ${ }^{4}$, S. Y. Eom ${ }^{5} .{ }^{1}$ Chungbuk National University Hospital, Internal Medicine, Division of Rheumatology, Cheongju-si, Chungcheongbuk-do, Korea, Republic of (South Korea); ${ }^{2}$ Chungbuk National University Hospital, Psychiatry, Cheongju, Korea, Republic of (South Korea); ${ }^{3}$ Chungbuk National University, Center for Environmental Medicine, Cheongju, Korea, Republic of (South Korea); ${ }^{4}$ Chungbuk National University College of Medicine, Division of Rheumatology, Department of Internal Medicine, Cheongju, Korea, Republic of (South Korea); ${ }^{5}$ College of Medicine, Chungbuk National University, Department of Preventive Medicine, Cheongju, Korea, Republic of (South Korea)

Background: Dementia is a common mental illness associated with aging Alzheimer's disease $(\mathrm{AD})$ and Vascular dementia $(\mathrm{VaD})$ are the most common causes of dementia in the elderly. In previous studies, hyperuricemia was suggested to have anti-oxidant effects and possible neuroprotective effects $[1,2]$.

Objectives: Hyperuricemia is the most important factor in the pathogenesis of gout. This study aims to investigate association between gout and dementia.

Methods: We conducted a nationwide population-based study using the NHISNSC database of the Korean populations consisted of 1 million individuals between 2002 and 2013. Gout patients were defined by International Classification of Diseases (ICD)-10 code for gout (M10) and prescriptions for urate lowering therapy including allopurinol, febuxostat and benzbromarone at least 30days. Dementia was also defined by ICD-10 codes for dementia; Alzheimer's disease (AD; F00), vascular dementia (VaD; F01). 